good preventive behaviours and have done their research, choosing to take a high-quality daily multivitamin is no cause for concern. The bigger picture here is that they want to protect themselves against future illness. We're in the second year of the pandemic and people feeling motivated to take control of their health is one - perhaps the only - positive to come out of this time.

\section{The context of poor nutrition}

We must also look at the context of long-term concerns about poor diets in the UK. Despite ongoing public health campaigns, the data for childhood and adult obesity, including hospital admissions where obesity was a factor, show a continued increase, year-on-year, particularly in deprived areas. ${ }^{8}$

Better nutrition means better health. It stabilises mood and improves immunity. ${ }^{9}$ Plenty of everyday foods are nutrient rich and, when part of a balanced diet, will give your patients what they need without having to take a supplement (unless there is a recommendation that they do).

A healthy diet is not boring. The typical Mediterranean diet - which includes fruit, vegetables and wholegrain cereals, also

\section{'Diet and nutrition must be discussed}

\section{[with patients]. If the conversation}

\section{turns to vitamins and supplements,} ensure you have plenty of resources"

variety of oral health disorders, particularly in children. These include increased risk of and onset of the progression of dental caries, higher prevalence of periodontitis and, in some cases, oral cancers. ${ }^{7}$ A connection can also be found between vitamin A and the regulation of salivary secretions, meaning patients lacking in vitamin A could be at higher risk of caries and gum disease due to xerostomia.

Research suggests that, for the most part, it is more beneficial to acquire these essential vitamins and minerals through dietary means. During your oral health consultations, diet and nutrition must be discussed. If the conversation turns to vitamins and supplements, and your patient is not in one of the groups for whom they are strongly advised, ensure you have plenty of resources on hand.

We have to consider the issue of choice. For example, if an individual is interested in their health and wellbeing, they adhere to moderate amounts of animal/fish protein and unsaturated fats - doesn't exclude the odd sweet treat or glass of something alcoholic and yet it is widely endorsed as the best way of eating to prevent disease, and even enjoy better mental wellbeing. ${ }^{10,11}$

\section{A common sense approach}

If people are choosing to take vitamins and supplements, this should be part of a commonsense approach to preventive health, alongside other proven practices. These include eating a balanced diet, daily exercise, drinking in moderation, avoiding tobacco and having an effective oral hygiene routine - TANDEX has daily toothbrushes, interdental brushes and other adjuncts that make this job simple.

The vitamins and supplement market is worth millions, and we must hope that this means more people than ever want to stay well.
By helping our patients learn as much as they can about the role of nutrition in preventing disease, and the other simple lifestyle changes that they can make, we will achieve better engagement, which will ultimately lead to improved outcomes for oral and general health.

For more information on Tandex's range of products, visit https://tandex.dk/.

\section{References}

1. Mintel Press Office. The Vitamin D Factor: Brits spend almost $£ 500$ million on vitamins and supplements. 23 October 2020. Available at: https://www.mintel.com/press-centre/ beauty-and-personal-care/the-vitamin-dfactor-brits-spend-almost-500-million-onvitamins-and-supplements (accessed March 2021).

2. Vimaleswaran K S, Forouhi N G, Khunti K. Vitamin D and covid-19. BMJ 2021; 372: n544.

3. NICE. COVID-19 rapid guideline: vitamin D. NICE guideline [NG187]. 17 December 2020. Available at: https://www.nice.org.uk/ guidance/ng187 (accessed March 2021).

4. NHS. Vitamin D. Available at: https://www. nhs.uk/conditions/vitamins-and-minerals/ vitamin-d/ (accessed March 2021).

5. NHS. Do I need vitamin supplements? Available at: https://www.nhs.uk/commonhealth-questions/food-and-diet/do-i-needvitamin-supplements/ (accessed March 2021).

6. BDA. The Association of UK Dietitians. Supplements: Food Fact Sheet. Available at: https://www.bda.uk.com/resource/ supplements.html (accessed March 2021).

7. Botelho J, Machado V, Proença L, Delgado A S, Mendes J J. Vitamin D deficiency and oral health: a comprehensive review. Nutrients 2020; 12: 1471. doi: 10.3390/nu12051471.

8. NHS Digital. Statistics on Obesity, Physical Activity and Diet, England, 2020. 5 May 2020. Available at: https://digital.nhs.uk/dataand-information/publications/statistical/ statistics-on-obesity-physical-activity-anddiet/england-2020 (accessed March 2021).

9. Chandra R K. Nutrition and the immune system from birth to old age. Eur J Clin Nutr 2002; 56: S73-76.

10. BBC. Ultra-processed food linked to early death. 30 May 2019. Available at: https:// www.bbc.co.uk/news/health-48446924 (accessed March 2021).

11. Firth J, Gangwisch J E, Borisini A, Wootton R E, Mayer E A. Food and mood: how do diet and nutrition affect mental wellbeing? BMJ 2020; 369: m2382. doi: 10.1136/bmj. $\mathrm{m} 2382$.

https://doi.org/10.1038/s41407-021-0644-6 